\title{
VELOCIDADE ECONÔMICA DE ESCOAMENTO E CUSTOS DE ENERGIA DE BOMBEAMENTO
}

\section{BRUNO L. T. PERRONI ${ }^{1}$, JACINTO DE A. CARVALHO ${ }^{2}$, LESSANDRO C. FARIA ${ }^{3}$}

RESUMO: Diversos fatores podem atuar como limitante para a atividade agrícola irrigada, dentre os quais a energia utilizada no sistema de irrigação tem grande destaque. Ainda que o sistema esteja hidraulicamente correto, ele pode não estar sendo economicamente viável para o produtor. Neste trabalho, foram feitas simulações para a determinação do diâmetro mais econômico para adutoras de aço galvanizado e PVC. Também foram obtidos modelos para a determinação dos custos de bombeamento. Foi constatado que, para tubulações de aço galvanizado, a velocidade econômica variou entre 0,67 e 1,63 $\mathrm{m} \mathrm{s}^{-1}$ e para PVC variou entre 0,62 e 1,97 $\mathrm{m} \mathrm{s}^{-1}$. Com os modelos gerados neste trabalho, é possível obter de forma mais rápida os parâmetros econômicos que envolvem os sistemas de bombeamento.

PALAVRA-CHAVE: diâmetro econômico, energia de bombeamento, tubulação.

\section{ECONOMIC SPEED FLOW AND PUMPING ENERGY COST}

\begin{abstract}
Several factors can act as limiting for irrigated agricultural activity, including energy used in the systems. Although the system is hydraulically correct this may not be economically feasible for the farmer. In this study simulations were made for determining the most economical diameter to galvanized steel and PVC pipelines. Models are also obtained to determine the cost of pumping. It was found that for galvanized steel pipes the economic speed ranged between 0.67 and $1.63 \mathrm{~ms}^{-1}$ and for PVC ranged between 0.62 and $1.97 \mathrm{~m} \mathrm{~s}^{-1}$. With the models generated in this study it can be achieved faster economic parameters that involve pumping systems.
\end{abstract}

KEYWORDS: diameter economic, energy pumping, pipes.

\section{INTRODUÇÃO}

A ampla utilização da irrigação como acréscimo tecnológico ressalta a importância do uso racional de recursos como água e energia elétrica. Diversos estudos são realizados no Brasil visando à maior eficiência nos projetos de irrigação, principalmente quando relacionados com a parte hidráulica do sistema. Estes estudos buscam melhor aproveitamento da água e da energia utilizada durante a irrigação.

Contudo, ainda que o sistema esteja hidraulicamente correto, ele pode não estar sendo economicamente viável para o produtor. Fatores como custo da energia, cultura e época de produção podem atuar como limitantes na agricultura irrigada. Torna-se, então, extremamente necessário um estudo mais aprofundado das variáveis econômicas que envolvem o projeto, principalmente quando relacionadas ao consumo de energia.

A tomada de decisão deve ser baseada em parâmetros econômicos atualizados, principalmente no mundo globalizado, onde a eficiência e os custos passam a ser os fatores de decisão. Assim, a utilização de velocidades e de diâmetros econômicos constitui ferramenta para a otimização dos custos dos sistemas de irrigação, devendo a seleção desses ser feita com base nas condições atuais da economia, com o objetivo de maximizar a eficiência de um sistema de recalque, ao mesmo tempo em que se procura minimizar os custos (CARVALHO \& REIS, 2000).

\footnotetext{
${ }^{1}$ Eng$^{\circ}$ Agrícola, Mestre em Engenharia Agrícola, UFLA, Lavras - MG. bruno.perroni@gmail.com.

${ }^{2}$ Professor Associado III - Departamento de Engenharia, UFLA, Lavras - MG, Bolsista CNPq. jacintoc@ufla.br.

${ }^{3}$ Eng $^{\circ}$ Agrícola, Doutor em Engenharia Agrícola, UFLA, Lavras - MG. lessandrofaria@ yahoo.com.br.

Recebido pelo Conselho Editorial em: 27-5-2008

Aprovado pelo Conselho Editorial em: 6-3-2011
} 
Segundo ZOCOLER et al. (2006), quando se utilizam em bombeamentos menores velocidades do fluxo, os diâmetros são maiores, o que proporciona maiores custos com tubulação e menores custos com bombas e motores. Os autores ainda apresentaram que a utilização de um bombeamento com tubulação de $0,250 \mathrm{~m}$, quando o diâmetro econômico deveria ser $0,284 \mathrm{~m}$, pode acarretar um consumo extra de energia elétrica de $30.800 \mathrm{kWh}$ por ano.

O dimensionamento econômico de uma tubulação é estabelecido quando, para cada condição, o diâmetro é determinado de forma a reduzir ao máximo o somatório do custo fixo com o custo anual variável (BERNARDO et al., 2006). Os autores ainda citam que existem diversas formas de se dimensionar economicamente uma tubulação.

De acordo com BATISTA \& COELHO (2003), todo o sistema de bombeamento deve ser projetado levando em conta critérios econômicos, uma vez que o diâmetro da tubulação, a potência do sistema de bombeamento e as despesas operacionais estão inter-relacionados.

A redução no diâmetro da tubulação eleva as perdas de carga e aumenta a potência necessária do conjunto motobomba; esta configuração de projeto propicia maior custo do conjunto elevatório e maior despesa com energia, embora proporcione economia na compra da tubulação. Por outro lado, aumentando-se o diâmetro da tubulação, a potência do conjunto elevatório e o custo operacional de energia elétrica serão menores, podendo tornar-se economicamente viável ao longo da vida útil dos equipamentos (LIMA et al., 2009).

De acordo com FAVETTA (1998), o diâmetro econômico é aquele que reduz ao mínimo a soma dos custos da tubulação e da energia. $\mathrm{O}$ autor ainda ressalta que é freqüente a necessidade de se estabelecer perdas de carga com base em diâmetros econômicos.

É de fundamental importância escolher o conjunto motobomba e a tubulação com base em critérios econômicos e não somente em critérios hidráulicos devido ao elevado investimento e ao custo de operação de um sistema de irrigação (CARVALHO et al., 2000).

Para a determinação do diâmetro utilizado em sistemas de bombeamento e, consequentemente, a perda de carga no sistema, são preferidas por projetistas as equações empíricas, como Hazen-Willians, Manning e Scobey em vez de utilizar a equação teórica de DarcyWeisbach. Entretanto, uma importante limitação das equações empíricas é o fato de um fator de rugosidade constante ser assumido para todas as velocidades de escoamento (KAMAND, 1998). O fato de essa suposição ser utilizada nas equações empíricas pode fazer com que a perda de carga difira da calculada pela equação de Darcy-Weisbach, que considera o fator de rugosidade variando conforme as condições do escoamento (BOMBARDELLI \& GARCÍA, 2003). Essa diferença pode influenciar na seleção do diâmetro a ser utilizado, e consequentemente, na determinação do diâmetro mais econômico e na estimativa dos gastos energéticos no bombeamento.

Em seu trabalho, LIMA et al. (2006) desenvolveram indicadores de eficiência energética para avaliar o aproveitamento de água e energia na irrigação. Os autores perceberam a ampla necessidade de avaliação de parâmetros, como o diâmetro utilizado na adutora, tempo de uso e tarifa de energia, pois o seu acompanhamento na fase de projeto e manutenção pode auxiliar o agricultor, tornando os equipamentos mais eficientes.

Sendo assim, o presente trabalho teve como objetivos a determinação da velocidade econômica de escoamento, em função das variáveis: vazão, comprimento da adutora e o material utilizado na mesma, tempo de funcionamento e desnível geométrico.

\section{MATERIAL E MÉTODOS}

A fim de se realizar este estudo, foi feito um levantamento, junto a diversos estabelecimentos comerciais, dos preços de cada componente do sistema de recalque, visando a gerar um preço médio para os mesmos. Nesse levantamento, foi considerado o preço na condição de pagamento à 
vista e com impostos incluídos. Visando a minimizar a influência dos impostos estaduais, foram coletados preços em diferentes estados da federação, incluindo todos da região Sudeste.

Foram pesquisados os preços de reduções excêntricas e concêntricas, mangotes flexíveis com $6 \mathrm{~m}$ de comprimento, curva dupla, registro de gaveta, curva de saída com bujão de escorva, válvula de pé, acoplamento elástico para motores, chave de proteção e partida, tubulações de aço galvanizado e PVC, bombas hidráulicas, motores elétricos e base de ferro para motor elétrico. A coleta dos dados ocorreu entre o período de 4 de junho e 31 de agosto de 2007, e, nesse período, o dólar apresentou uma cotação média de $\mathrm{R} \$ 1,94$.

Foi definido um sistema de recalque padrão, composto de uma tubulação de sucção com válvula de pé, motor, bomba, entrada e saída da bomba, acoplamento e sistemas de partida e proteção.

Foram adotadas para o estudo as tubulações de aço galvanizado, para pressões de até $1.500 \mathrm{kPa}$ e tubulações de PVC nas quais a pressão de operação simulada foi menor que $125 \mathrm{kPa}$.

\section{Custo da energia de bombeamento}

Para a determinação do custo da energia de bombeamento, foram utilizadas diferentes situações de operação do sistema, envolvendo interações entre diferentes pressões de funcionamento, vazões, diâmetros e comprimentos de tubulação e tempos de funcionamento. $\mathrm{O}$ custo da energia (CE) pode ser representado pela relação abaixo, em que $\mathrm{Q}, \mathrm{H}_{\mathrm{man}}, \mathrm{T}_{\mathrm{f}}$ representam, respectivamente, vazão, altura manométrica e tempo de funcionamento:

$$
\mathrm{CE}=\mathrm{f}\left(\mathrm{Q}, \mathrm{H}_{\text {man }}, \mathrm{T}_{\mathrm{f}}\right)
$$

Foram analisados diferentes diâmetros e vazões, utilizando valores que atendessem às situações mais comumente encontradas nos projetos de irrigação. Analisaram-se diâmetros de 50; $75 ; 100 ; 125 ; 150 ; 200 ; 250 ; 300$ e $350 \mathrm{~mm}$ e vazões de 0,00138 a $0,0833 \mathrm{~m}^{3} \mathrm{~s}^{-1}$.

\section{Altura manométrica total}

A altura manométrica foi determinada pelo somatório do valor da pressão necessária no início da área irrigada (Pin), desnível geométrico total $(\mathrm{Hg})$ e perda total nas tubulações de sucção $\left(\mathrm{Hf}_{\mathrm{s}}\right)$ e recalque $\left(\mathrm{Hf}_{r}\right)$ :

$$
\mathrm{H}_{\text {man }}=\mathrm{Pin}+\mathrm{Hg}+\mathrm{Hf}_{\mathrm{s}}+\mathrm{Hf}_{\mathrm{r}}
$$

Utilizaram-se para representar a altura manométrica total das situações simuladas, valores variando de 500 a $1.500 \mathrm{kPa}$. Foi considerado como Pin o valor de pressão registrado no início da área a ser irrigada para o funcionamento do sistema, sendo essa com diferença de nível igual a zero, ou seja, plana. Para a perda de carga na sucção, foi adotado um valor médio de $5 \mathrm{kPa}$ (cerca de $0,5 \mathrm{~J} \mathrm{~N}^{-1}$ ). A perda de carga no recalque foi calculada utilizando a equação de Hazen-Williams. Aequação de Hazen-Williams foi utilizada devido ao fato de apresentar resultados consistentes quando utilizada com diâmetros maiores que $50 \mathrm{~mm}$ e a sua grande utilização em cálculos dessa natureza. Adotou-se um coeficiente de 125 para tubulações de aço galvanizado e de 140 para tubulações de PVC.

\section{Potência do sistema}

A potência do motor necessária para a relação entre vazão e pressão, chamada de potência absorvida do motor elétrico, foi calculada pela equação 3:

$$
\operatorname{Pot}_{\mathrm{a}}=\frac{\mathrm{Q} \mathrm{H}_{\operatorname{man}} \gamma}{1000 \eta}
$$


em que,

Pot $_{\mathrm{a}}$ - potência absorvida, $\mathrm{kW}$;

$\mathrm{Q}$ - vazão, $\mathrm{m}^{3} \mathrm{~s}^{-1}$;

$\mathrm{H}_{\text {man }}$ - altura manométrica, m;

$\gamma$ - peso específico da água, considerado aproximadamente $9.800 \mathrm{~N} \mathrm{~m}^{-3}, \mathrm{e}$

$\eta$ - rendimento do conjunto motobomba, decimal $\left(\eta=\eta_{m} \eta_{b}\right)$.

Para as bombas, os rendimentos variaram entre 40 e $75 \%$, conforme os valores encontrados nas curvas características fornecidas pelos fabricantes e para os motores elétricos de 80 a $95 \%$, o que resultou em um rendimento global médio de $60 \%$ para o conjunto motobomba.

Foi utilizado um acréscimo de potência, sendo esse uma margem de segurança para o funcionamento do motor elétrico. Esse acréscimo foi obtido em função do tipo de motor e da própria potência.

\section{Custos fixos}

O custo fixo total (CF) foi definido pela soma do custo de aquisição do sistema de recalque $\left(\mathrm{C}_{\mathrm{SR}}\right)$, tubulação $\left(\mathrm{C}_{\mathrm{T}}\right)$ e a depreciação $\left(\mathrm{C}_{\mathrm{Dep}}\right)$ destes durante sua vida útil. $\mathrm{O}$ custo do sistema de recalque foi dado pelo somatório dos valores do custo da sucção, motor elétrico com chaves de proteção/partida e bomba hidráulica. Com interações entre diferentes valores de vazão e pressão, obteve-se o custo total do sistema de recalque.

O custo da tubulação de sucção foi dado pelo somatório dos custos de um mangote flexível de 6 m, uma válvula de pé e uma redução excêntrica.

O custo do motor elétrico trifásico com sistema de proteção e acoplamento foi obtido por meio de um ajuste, feito por regressão. Foi gerado um preço médio entre motores e acoplamentos com rotação de 1.750 e $3.500 \mathrm{rpm}$ e outro preço médio para os sistemas de proteção de diferentes tensões.

No caso do custo das bombas existentes no mercado para atender às situações simuladas, foram utilizados catálogos técnicos fornecidos pelos fabricantes, selecionando-se nas curvas características de cada bomba o ponto de máximo rendimento e, desse, foram extraídos os valores de pressão e vazão. Adotou-se o ponto de máximo rendimento como sendo o ponto de operação da bomba. Utilizando valores de vazão, pressão e custo, foi ajustado um modelo que representasse o custo da bomba em função dos valores de vazão e pressão.

Para a determinação do custo da tubulação, foram analisadas separadamente as tubulações de aço galvanizado e de PVC.

O custo das tubulações de recalque foi determinado com base em diversas combinações simuladas de valores de comprimento e diâmetro de tubulação. Foram utilizados, para determinação do custo da tubulação de aço galvanizado nesse estudo, diâmetros variando de 50 a $350 \mathrm{~mm}$ e comprimentos até $2.000 \mathrm{~m}$. Com base em regressões com essas combinações de valores, foi possível obter o custo da tubulação de aço galvanizado. O custo das tubulações de PVC foi determinado da mesma forma; entretanto, os diâmetros analisados variaram entre 50 e $300 \mathrm{~mm}$. Para tubulações de PVC, foram geradas duas funções de custos, sendo uma para tubos com pressão nominal de $800 \mathrm{kPa}$ e outra para tubos com pressão nominal de $1.250 \mathrm{kPa}$.

Estes custos fixos foram anualizados, utilizando o fator de recuperação de capital (FRC) que leva em consideração a taxa de juros (i) e a vida útil ou tempo de amortização (n):

$$
F R C=\frac{(1+i)^{\mathrm{n}} i}{(1+\mathrm{i})^{\mathrm{n}}-1}
$$


Foi considerada uma taxa de juros de $12 \%$ a.a. Esse valor justifica-se pela estabilidade econômica do País nos últimos anos, além de representar um valor médio praticado ultimamente. A vida útil média dos componentes adotada para a determinação do fator de recuperação foi de 15 anos, conforme o PRONI (1987). O custo fixo anual foi dado pela multiplicação do fator de recuperação de capital pelo custo fixo total.

Não foram contabilizados, aqui, os valores de sucata dos equipamentos ao final do período analisado.

\section{Custos variáveis anuais}

Os custos variáveis foram definidos como sendo o somatório dos gastos com manutenção e energia elétrica utilizada para o funcionamento do sistema de irrigação.

Os gastos com manutenção foram calculados conforme PRONI (1987), em que esses representam até $2 \%$ do custo fixo total.

Para a determinação do custo da energia de bombeamento, foram utilizadas diferentes situações de operação do sistema, envolvendo interações entre diferentes pressões de funcionamento, vazões, diâmetros e comprimentos de tubulação e tempos de funcionamento.

O consumo de energia dos motores elétricos durante o um ano foi obtido relacionando a potência absorvida e o tempo de funcionamento do sistema. Adotaram-se tempos de funcionamento de $720 ; 1.440 ; 1.680$ e 2.880 e 3.600 horas por ano.

A determinação do custo da energia elétrica obedeceu aos critérios da RESOLUÇÃO ANEEL $\mathrm{N}^{\mathrm{o}}$ 456, de 29 de novembro de 2000, no Art. $2^{\circ}$, itens XXII e XIII, que classifica os consumidores por potência instalada em basicamente dois grupos. Os consumidores do grupo B, com transformadores de até $75 \mathrm{kVA}$, e consumidores do grupo A, com transformadores maiores que $75 \mathrm{kVA}$.

Para os cálculos do custo de energia dos consumidores do grupo A, a tarifação possui estruturas com dois componentes básicos na definição do seu preço, ou seja, o consumo de energia $(\mathrm{kWh})$ e a demanda de potência $(\mathrm{kWh})$. O sistema de tarifação utilizado foi o Horo-Sazonal verde que permitiu melhor aproveitamento dos benefícios oferecidos pela concessionária, especialmente entre os consumidores de médio porte, com demanda na faixa de $50 \mathrm{~kW}$ a $500 \mathrm{~kW}$.

Adotou-se, neste estudo, o funcionamento no período seco, quando existe a necessidade de se irrigar, e fora do período de ponta, evitando as tarifas elevadas dos horários de pico de consumo de energia.

O custo da energia para os consumidores do grupo A foi determinada por:

$$
\mathrm{CE}=\mathrm{D}_{\mathrm{c}} \mathrm{T}_{\mathrm{d}}+\mathrm{C}_{\text {efp }} \mathrm{T}_{\text {efp }} \mathrm{T}_{\mathrm{f}}
$$

em que,

CE- custo da energia, $\mathrm{R} \$$;

$\mathrm{D}_{\mathrm{c}}$ - demanda contratada, $\mathrm{kW}$;

$\mathrm{T}_{\mathrm{d}}$ - tarifa de demanda, $\mathrm{R} \$ \mathrm{~kW}^{-1}$;

$\mathrm{C}_{\text {efp }}$ - consumo de energia em horário fora de ponta, $\mathrm{kWh}$;

$\mathrm{T}_{\mathrm{efp}}$ - tarifa de consumo em horário fora de ponta, $\mathrm{R} \$ \mathrm{kWh}^{-1}$, e

$\mathrm{T}_{\mathrm{f}}$ - tempo de funcionamento, horas.

Foi utilizada, para o cálculo dos consumidores do grupo $\mathrm{B}$, a tarifa convencional, sendo o custo com energia dos consumidores do grupo B determinado pela seguinte equação:

$$
\mathrm{CE}=\mathrm{C}_{\mathrm{ee}} \mathrm{T}_{\mathrm{e}} \mathrm{T}_{\mathrm{f}}
$$


em que,

$\mathrm{CE}$ - custo da energia, $\mathrm{R} \$$

$\mathrm{C}_{\mathrm{ee}}$ - consumo de energia elétrica, $\mathrm{kWh}$;

$\mathrm{T}_{\mathrm{e}}$ - tarifa de energia, $\mathrm{R} \$ \mathrm{kWh}^{-1}$, e

$\mathrm{T}_{\mathrm{f}}$ - tempo de funcionamento, horas.

\section{Diâmetros e velocidades econômicas}

Considerou-se diâmetro econômico aquele em que, para uma mesma situação, foram mínimos os custos provenientes do bombeamento (incluindo amortização e custos variáveis) e os custos da própria tubulação (incluindo também a amortização e a manutenção).

Foram gerados valores de velocidade econômica média, divididos por faixa de vazões para tempos de funcionamento de $720 ; 1.440 ; 1.680 ; 2.880$ e 3.600 horas e comprimentos da tubulação de $100 ; 200 ; 500$ e $1.000 \mathrm{~m}$.

Foi possível obter, com base nas velocidades econômicas, os valores do coeficiente $\mathrm{K}$ da equação de Bresse.

\section{RESULTADOS E DISCUSSÃO}

Na sequência, serão apresentadas as equações ajustadas para a determinação dos custos do motor elétrico, bomba, tubulação de aço galvanizado, tubulações de PVC e sistema de recalque que foram analisados neste estudo.

Para a obtenção do custo do motor elétrico $\left(\mathrm{C}_{\mathrm{ME}}\right)$ em Reais, foi ajustada uma equação na qual o custo está em função da potência do motor (Pot) em cv:

$\mathrm{C}_{\mathrm{ME}}=152,37$ Pot +1149

$\mathrm{r}^{2}=0,988$

O modelo ajustado para o custo da bomba hidráulica em um sistema de recalque, em função da altura manométrica e da vazão, está apresentado na eq.(8):

$\mathrm{C}_{\mathrm{B}}=\mathrm{e}^{\left[7,43+0,008 \mathrm{H}_{\operatorname{man}}+812 \mathrm{Q}^{2,5}\right]}$

$\mathrm{r}^{2}=0,969$

em que,

$\mathrm{C}_{\mathrm{B}}$ - custo da bomba, $\mathrm{R} \$$

$\mathrm{H}_{\text {man }}$ - altura manométrica, $\mathrm{kPa}$, e

$\mathrm{Q}$ - vazão, $\mathrm{m}^{3} \mathrm{~s}^{-1}$.

Foi ajustado um modelo que permite obter o custo da tubulação de aço galvanizado:

$\mathrm{C}_{\mathrm{Tag}}=\mathrm{e}^{\left[1,43+\ln (\mathrm{L})+6,10(\mathrm{D})^{0,5}\right]}$

$\mathrm{r}^{2}=0,997$

em que,

$\mathrm{C}_{\text {Tag }}$ - custo da tubulação de aço galvanizado, $\mathrm{R} \$$;

$\mathrm{L}$ - comprimento da tubulação, m, e

D - diâmetro da tubulação, m. 
Para tubulação de PVC, foram obtidas duas equações: uma para pressão menor que $800 \mathrm{kPa}$ [eq.(10)] e, outra, para pressão até $1.250 \mathrm{kPa}$ [eq.(11)]:

$$
\mathrm{C}_{\mathrm{TPVC}_{80}}=\mathrm{e}^{\left[-0,4563+\ln (\mathrm{L})+8,62(\mathrm{D})^{0,5}\right]}
$$

$$
\mathrm{r}^{2}=0,986
$$

em que,

$\mathrm{C}_{\mathrm{TPVC} 80}$ - custo da tubulação de PVC PN80, R\$;

L- comprimento da tubulação, $\mathrm{m}, \mathrm{e}$

D - diâmetro da tubulação, $\mathrm{m}$.

$$
\mathrm{C}_{\mathrm{TPVC}_{125}}=\mathrm{e}^{\left[0,238+\ln (\mathrm{L})+7,844(\mathrm{D})^{0,5}\right]}
$$

$$
\mathrm{r}^{2}=0,997
$$

em que,

$\mathrm{C}_{\mathrm{TPVC}_{125}}$ - custo da tubulação de PVC PN125, R\$;

L - comprimento da tubulação, $\mathrm{m}, \mathrm{e}$

$\mathrm{D}$ - diâmetro da tubulação, $\mathrm{m}$.

O custo do sistema de recalque (incluindo motor, bomba, acoplamento, chaves de proteção e partida, sucção e ligação de pressão) pode ser estimado pela eq.(12):

$$
\mathrm{C}_{\text {sr }}=\mathrm{e}^{\left[6,62+0,054 \mathrm{Hman}^{0,5}+9,12 \mathrm{Q}^{0,5}\right]}
$$

$$
r^{2}=0,993
$$

em que,

$\mathrm{C}_{\mathrm{sr}}$ - custo do sistema de recalque, $\mathrm{R} \$$;

$\mathrm{H}_{\text {man }}$ - altura manométrica, $\mathrm{m}$, e

$\mathrm{Q}$ - vazão, $\mathrm{m}^{3} \mathrm{~s}^{-1}$.

Os valores de velocidade econômica e da constante $\mathrm{K}$ da equação de Bresse, em função do comprimento da adutora, da vazão do sistema e do tempo de funcionamento, estão apresentados nas Tabelas 1 e 2 para tubulações de aço galvanizado, e nas Tabelas 3 e 4 para PVC.

TABELA 1. Velocidade econômica para adutoras de aço galvanizado com comprimentos de 100; 500 e $1.000 \mathrm{~m}$ e tempo de funcionamento de 1.440 horas por ano. Economic speed for galvanized steel pipelines with lengths of 100,500 and $1000 \mathrm{~m}$ and running time of 1440 hours per year.

\begin{tabular}{ccccccc}
\hline \multirow{2}{*}{$\mathrm{Q}\left(\mathrm{m}^{3} \mathrm{~s}^{-1}\right)$} & \multicolumn{7}{c}{1.440 horas ano $^{-1}$} \\
\cline { 2 - 7 } & \multicolumn{2}{c}{$100 \mathrm{~m}$} & \multicolumn{2}{c}{$500 \mathrm{~m}$} & \multicolumn{2}{c}{$1.000 \mathrm{~m}$} \\
\cline { 2 - 7 } & $\mathrm{V}\left(\mathrm{m} \mathrm{s}^{-1}\right)$ & $\mathrm{K}$ & $\mathrm{V}\left(\mathrm{m} \mathrm{s}^{-1}\right)$ & $\mathrm{K}$ & $\mathrm{V}\left(\mathrm{m} \mathrm{s}^{-1}\right)$ & $\mathrm{K}$ \\
\hline$<0,011$ & 0,71 & 1,34 & 0,71 & 1,34 & 0,71 & 1,34 \\
$0,011-0,022$ & 1,07 & 1,09 & 1,07 & 1,09 & 1,26 & 1,01 \\
$0,022-0,033$ & 1,05 & 1,10 & 1,28 & 1,00 & 1,28 & 1,00 \\
$0,033-0,044$ & 1,28 & 1,00 & 1,28 & 1,00 & 1,46 & 0,93 \\
$0,044-0,055$ & 1,43 & 0,94 & 1,43 & 0,94 & 1,43 & 0,94 \\
$0,055-0,066$ & 1,37 & 0,96 & 1,37 & 0,96 & 1,37 & 0,96 \\
$0,066-0,083$ & 1,45 & 0,94 & 1,39 & 0,96 & 1,48 & 0,93 \\
\hline Média & 1,19 & 1,03 & 1,22 & 1,02 & 1,28 & 1,00 \\
\hline
\end{tabular}


TABELA 2. Velocidade econômica para adutoras de aço galvanizado com comprimentos de 100; 500 e $1.000 \mathrm{~m}$ e tempo de funcionamento de 2.880 horas por ano. Economic speed for galvanized steel pipelines with lengths of 100,500 and $1000 \mathrm{~m}$ and running time of 2880 hours per year.

\begin{tabular}{|c|c|c|c|c|c|c|}
\hline \multirow{3}{*}{$\mathrm{Q}\left(\mathrm{m}^{3} \mathrm{~s}^{-1}\right)$} & \multicolumn{6}{|c|}{2.880 horas no $^{-1}$} \\
\hline & \multicolumn{2}{|c|}{$100 \mathrm{~m}$} & \multicolumn{2}{|c|}{$500 \mathrm{~m}$} & \multicolumn{2}{|c|}{$1.000 \mathrm{~m}$} \\
\hline & $\mathrm{V}\left(\mathrm{m} \mathrm{s}^{-1}\right)$ & $\mathrm{K}$ & $\mathrm{V}\left(\mathrm{m} \mathrm{s}^{-1}\right)$ & $\mathrm{K}$ & $\mathrm{V}\left(\mathrm{m} \mathrm{s}^{-1}\right)$ & $\mathrm{K}$ \\
\hline$<0,011$ & 0,69 & 1,36 & 0,69 & 1,36 & 0,69 & 1,36 \\
\hline $0,011-0,022$ & 0,89 & 1,20 & 0,89 & 1,20 & 0,89 & 1,20 \\
\hline $0,022-0,033$ & 0,94 & 1,16 & 0,94 & 1,16 & 0,94 & 1,16 \\
\hline $0,033-0,044$ & 1,13 & 1,06 & 0,97 & 1,15 & 0,97 & 1,15 \\
\hline $0,044-0,055$ & 1,04 & 1,11 & 1,04 & 1,11 & 1,04 & 1,11 \\
\hline $0,055-0,066$ & 1,37 & 0,96 & 1,26 & 1,00 & 1,26 & 1,00 \\
\hline $0,066-0,083$ & 1,18 & 1,04 & 1,03 & 1,11 & 1,03 & 1,11 \\
\hline Media & 1,03 & 1,11 & 0,98 & 1,14 & 0,98 & 1,14 \\
\hline
\end{tabular}

TABELA 3. Velocidade econômica para adutoras de PVC com comprimentos de 100; 500 e $1.000 \mathrm{~m}$ e tempo de funcionamento de 1.440 horas por ano. Economic speed for PVC pipelines with lengths of 100,500 and $1000 \mathrm{~m}$ and running time of 1440 hours per year.

\begin{tabular}{|c|c|c|c|c|c|c|}
\hline \multirow{3}{*}{$\mathrm{Q}\left(\mathrm{m}^{3} \mathrm{~s}^{-1}\right)$} & \multicolumn{6}{|c|}{1.440 horas ano $^{-1}$} \\
\hline & \multicolumn{2}{|c|}{$100 \mathrm{~m}$} & \multicolumn{2}{|c|}{$500 \mathrm{~m}$} & \multicolumn{2}{|c|}{$1.000 \mathrm{~m}$} \\
\hline & $\mathrm{V}\left(\mathrm{m} \mathrm{s}^{-1}\right)$ & $\mathrm{K}$ & $\mathrm{V}\left(\mathrm{m} \mathrm{s}^{-1}\right)$ & $\mathrm{K}$ & $\mathrm{V}\left(\mathrm{m} \mathrm{s}^{-1}\right)$ & $\mathrm{K}$ \\
\hline$<0,011$ & 0,65 & 1,40 & 0,65 & 1,40 & 0,65 & 1,40 \\
\hline $0,011-0,022$ & 0,98 & 1,14 & 0,98 & 1,14 & 0,98 & 1,14 \\
\hline $0,022-0,033$ & 1,05 & 1,10 & 1,05 & 1,10 & 1,05 & 1,10 \\
\hline $0,033-0,044$ & 1,20 & 1,03 & 1,20 & 1,03 & 1,46 & 0,93 \\
\hline $0,044-0,055$ & 1,43 & 0,94 & 1,43 & 0,94 & 1,43 & 0,94 \\
\hline $0,055-0,066$ & 1,37 & 0,96 & 1,37 & 0,96 & 1,37 & 0,96 \\
\hline $0,066-0,083$ & 1,53 & 0,91 & 1,53 & 0,91 & 1,53 & 0,91 \\
\hline Media & 1,17 & 1,04 & 1,17 & 1,04 & 1,21 & 1,03 \\
\hline
\end{tabular}

TABELA 4. Velocidade econômica para adutoras de PVC com comprimentos de 100; 500 e $1.000 \mathrm{~m}$ e tempo de funcionamento de 2.880 horas por ano. Economic speed for PVC pipelines with lengths of 100,500 and $1000 \mathrm{~m}$ and running time of 2880 hours per year.

\begin{tabular}{|c|c|c|c|c|c|c|}
\hline \multirow{3}{*}{$\mathrm{Q}\left(\mathrm{m}^{3} \mathrm{~s}^{-1}\right)$} & \multicolumn{6}{|c|}{2880 horas ano $^{-1}$} \\
\hline & \multicolumn{2}{|c|}{$100 \mathrm{~m}$} & \multicolumn{2}{|c|}{$500 \mathrm{~m}$} & \multicolumn{2}{|c|}{$1.000 \mathrm{~m}$} \\
\hline & $\mathrm{V}\left(\mathrm{m} \cdot \mathrm{s}^{-1}\right)$ & $\mathrm{K}$ & $\mathrm{V}\left(\mathrm{m} \cdot \mathrm{s}^{-1}\right)$ & $\mathrm{K}$ & $\mathrm{V}\left(\mathrm{m} \cdot \mathrm{s}^{-1}\right)$ & $\mathrm{K}$ \\
\hline$<0,011$ & 0,62 & 1,43 & 0,62 & 1,43 & 0,62 & 1,43 \\
\hline $0,011-0,022$ & 0,80 & 1,26 & 0,80 & 1,26 & 0,80 & 1,26 \\
\hline $0,022-0,033$ & 0,94 & 1,16 & 0,94 & 1,16 & 0,94 & 1,16 \\
\hline $0,033-0,044$ & 1,13 & 1,06 & 1,13 & 1,06 & 1,39 & 0,96 \\
\hline $0,044-0,055$ & 1,04 & 1,11 & 1,04 & 1,11 & 1,14 & 1,06 \\
\hline $0,055-0,066$ & 1,26 & 1,01 & 1,26 & 1,01 & 1,37 & 0,96 \\
\hline $0,066-0,083$ & 1,32 & 0,98 & 1,27 & 1,00 & 1,32 & 0,98 \\
\hline Media & 1,02 & 1,12 & 1,01 & 1,12 & 1,08 & 1,08 \\
\hline
\end{tabular}

Os valores de velocidades médias encontrados entre as diversas situações analisadas variaram entre 0,67 e 1,63 $\mathrm{m} \mathrm{s}^{-1}$ para tubulações de aço galvanizado e entre 0,62 e 1,97 $\mathrm{m} \mathrm{s}^{-1}$ para tubulações de PVC, sendo esses valores diferentes dos apresentados nas diversas literaturas existentes, que consideram velocidades para o dimensionamento de até $2,4 \mathrm{~m} \mathrm{~s}^{-1}$, como apresentado por AZEVEDO NETO et al. (2002) e BERNARDO et al. (2006). 
As oscilações (descontinuidade da tendência de variação) observadas para os valores de velocidade econômica nas Tabelas 1 a 4 ocorrem devido à progressiva alteração do diâmetro à medida que se muda a vazão.

Analisando-se um mesmo tipo de material da tubulação e tempo de funcionamento, observase que o comprimento da tubulação não apresentou influência sobre o valor final da velocidade econômica, ou seja, esta praticamente permaneceu inalterada, independentemente do comprimento da adutora. A variação do comprimento da adutora reflete diretamente nos custos (fixos e variáveis), ou seja, quanto maior o comprimento maior o custo fixo, e, também, maiores os custos variáveis (maior perda de carga e consequentemente maior consumo). Assim, o aumento dos custos fixos, no presente estudo, é equilibrado com aumento dos custos variáveis, redundando na permanência do mesmo diâmetro econômico, independentemente dos valores de comprimento analisados.

Considerando um mesmo tipo de material da tubulação, observa-se, entre as Tabelas 1 e 2 para aço galvanizado, e 3 e 4 para PVC, que a velocidade econômica varia de forma inversa ao tempo de funcionamento, ou seja, à medida que se aumenta o tempo de funcionamento, a velocidade média econômica tende a diminuir. Maior tempo de funcionamento representa maior gasto com energia e, consequentemente, maior custo variável, fazendo com que o diâmetro econômico seja maior, e o valor da velocidade, menor.

A amplitude dos valores de velocidade econômica apresentados neste trabalho estão abaixo daqueles normalmente indicados na literatura (dimensionamento utilizando velocidades entre $0,8 \mathrm{e}$ $2,5 \mathrm{~m} \mathrm{~s}^{-1}$ ) e, também, daqueles utilizados em muitos projetos técnicos.

\section{CONCLUSÕES}

Para tubulação de aço galvanizado, a velocidade econômica varia entre $0,67 \mathrm{~m} \mathrm{~s}^{-1}$ e $1,63 \mathrm{~m} \mathrm{~s}^{-1}$ e para PVC, varia entre 0,62 e $1,97 \mathrm{~m} \mathrm{~s}^{-1}$. diminuir.

Com o aumento do tempo de funcionamento do sistema, a velocidade econômica tende a

\section{REFERÊNCIAS}

AZEVEDO NETTO, J.M.; FERNANDES, M.F.; ARAUJO, R.; ITO, A.E. Manual de hidráulica. 8.ed. São Paulo: E. Blücher, 2002. 669 p.

BATISTA, M.B.; COELHO, M.M.L.P. Fundamentos de engenharia hidráulica. Belo Horizonte, UFMG, 2003. $440 \mathrm{p}$.

BERNARDO, S.; SOARES, A.A.; MANTOVANI, E.C. Manual de irrigação. 8.ed. Viçosa. Ed: UFV, 2006. 625 p.

BOMBARDELLI, F.A.; GARCÍA, H. Hydraulic design of large-diameter pipes. Journal of Hydraulics Engineering, New York, v.129, n.11, p.839-846, 2003.

CARVALHO, J.A.; BRAGA JÚNIOR, R.A.; REIS, J.B.R.S. Análise de custos na escolha do tipo de motor para acionamento de bombas em áreas irrigadas. Ciência e Agrotecnologia, Lavras, v.24, n.2, p.434-440, 2000.

CARVALHO, J.A.; REIS, J.B.R.S. Avaliação dos custos de energia de bombeamento e determinação do diâmetro econômico da tubulação. Ciência e Agrotecnologia, Lavras, v.24, n.2, p.411-449, 2000.

FAVETTA, G.M. Estudo Econômico do sistema de adução em equipamentos de irrigação do tipo pivô central. 1998. 110 f. Tese (Doutorado em Agronomia) - Escola Superior de Agricultura "Luiz de Queiroz", Universidade de São Paulo, Piracicaba, 1998. 
KAMAND, F.Z. Hydraulic friction factors for pipe flow. Journal of Irrigation and Drainage Engineering, New York, v.114, n.2, p.311-323, 1998.

LIMA, A.C.; GUIMARÃES JÚNIOR, S.C.; FIETZ, C.R; CAMACHO, J.R. Avaliação e análise da eficiência energética na irrigação em sistemas de pivô central. Revista Brasileira de Engenharia Agrícola e Ambiental, v.13, n.4, jul./ago., 2009.

LIMA, A.C.; GUIMARÃES JÚNIOR, S.C.; CAMACHO, J.R.; SALERNO, C.H. Desenvolvimento de indicadores de eficiência energética para avaliação de pivôs centrais de irrigação. In:

CONGRESSO BRASILEIRO DE ENGENHARIA AGRÍCOLA, 35., 2006, João Pessoa. Anais... Jaboticabal: SBEA, 2006. 1 CD-ROM.

PRONI. PROGRAMA NACIONAL DE IRRIGAÇÃO. Tempo de irrigar: manual do irrigante. São Paulo: Mater, Fundação Victor Civita, 1987. 160 p.

ZOCOLER, J.L.; BAGGIO FILHO, F.C.; OLIVEIRA, L.A.F.; HERNANDEZ, F.B.T. Model for determining flow diameter and economic velocity in water elevating systems. Mathematical Problems in Engineering, Nasr City, v.2006, p.1-17, 2006. 